\title{
Enforcing Point-wise Priors on Binary Segmentation
}

\section{Feng Li}

http://www.fengl.org/

Fatih Porikli

http://www.porikli.com/
Light

Palo Alo, CA 94301, USA

Australian National University / NICTA

Canberra ACT 0200, Australia
Segmentation is one of the extensively studied areas in computer vision. Pioneering work [4] treats image segmentation as a graph partitioning problem and proposes a normalized cut criterion measures both the total dissimilarity between the different groups as well as the total similarity within the groups. Seminal work [3] derives a matting Laplacian matrix from multiple matte equations. In comparison with random walk [2] and normalized cuts [4], they adapt a correlation measure instead of the exponent of color distance, a local scaling instead of a global scaling, and formulate a least-squares solution with constraints from user input. Local scaling leads to better clustering especially when the data includes multiple scales and the clusters are placed within a cluttered background.

Non-negative point-wise priors such as saliency map, defocus field, foreground mask, object location window, and user given seeds, come in the form of confidence or probability values, and they are often incomplete, irregular, and noisy, which eventually makes the labelling task a challenge. In this paper, we aim to to extract image regions that are aligned on the object boundaries and also in accordance with the given point-wise priors. To this end, we define a graph Laplacian spectrum based cost function and embed it into a minimization framework. For a comprehensive understanding, we analyze five alternative formulations, and demonstrate that the robust function version produces consistently superior results.

To estimate the optimal $\mathbf{x}$ from the prior $\mathbf{x}^{\star}$, we compute a graph Laplacian matrix $L$ from $G$, where $G$ is a graph representation of the input image $\mathbf{y}$. In other words, the Laplacian matrix $L$ will regularize our underconstrained optimization formulation by laying on the image structure inherent in $\mathbf{y}$. This enables us to define the binary segmentation problem as a least-squares constrained optimization

$$
\min _{\mathbf{x}}\left\|\mathbf{x}-\mathbf{x}^{\star}\right\|^{2}, \quad \text { s.t. } \quad L \mathbf{x}=0 .
$$

We call the above constraint $L \mathbf{x}=0$ the Laplacian spectrum constraint. This is a generalization of the conventional approaches and does not require a specific numerical solver as the matting Laplacian. This constraint enforces a given image structure on the prior information (in the data fidelity term $\left\|\mathbf{x}-\mathbf{x}^{\star}\right\|^{2}$ ). With this constraint, the optimal $\mathbf{x}$ should lie in the null-space of $L$, which means $\mathbf{x}$ should be constant within each connected component of the graph $G$.

Depnding on the norm used for the Laplacian spectrum constraint, we define 5 alternative objective functions solving the optimal $\mathbf{x}$. Here we only discuss about Convex Function and Robust Function with $\ell_{2}$ Norm on Constraint, for the rest 3 formations please refer to our paper.

Instead of solving a constrained optimization problem Eq.(1), we can transform it into an unconstrained minimization:

$$
\min _{\mathbf{x}}\left\|\mathbf{x}-\mathbf{x}^{\star}\right\|^{2}+\beta\|L \mathbf{x}\|^{2},
$$

with a penalty $\beta$ that enforces the structure in $\mathbf{y}$. Setting the derivative of the objective function Eq.(2) to 0, we obtain a closed form solution:

$$
\mathbf{x}=\left(\beta L^{\top} L+I\right)^{-1} \mathbf{x}^{\star}=P \mathbf{x}^{\star},
$$

where $I$ is an identity matrix, and $P$ can be viewed as a modified projection matrix $^{1}$.

Since the residual $\delta=\left|\mathbf{x}-\mathbf{x}^{\star}\right|$ has many spatially continuous large outliers and the least square data fidelity term weights each sample with a quadratic norm, the final estimation of Eq.(2) can be distorted severely. Depending on its quality, the prior information $\mathbf{x}^{\star}$ could contain incomplete and inaccurate indicators, for instance strong responses across segment boundaries. This may confuse the segmentation algorithm and cause mislabeling. A better option is to weight large outliers less and use the structure information from the Laplacian spectrum constraint to recover

${ }^{1} P$ does not satisfy the idempotent property of the projection matrix

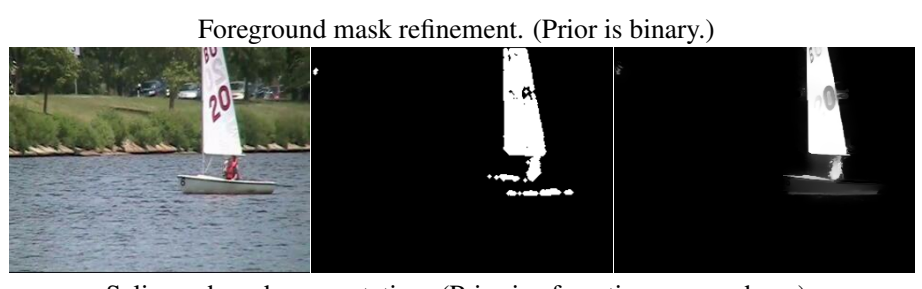

Saliency based segmentation. (Prior is of continuous numbers.)

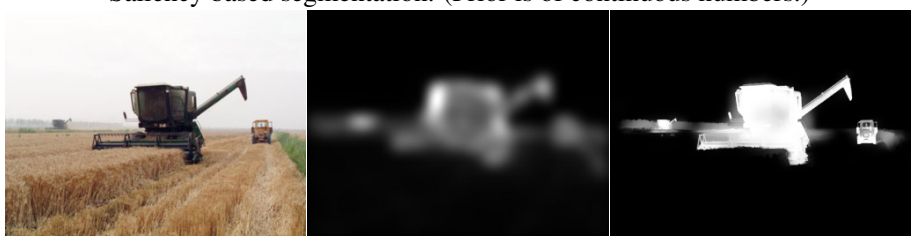

Infocus region segmentation. (Prior is of continuous numbers.)

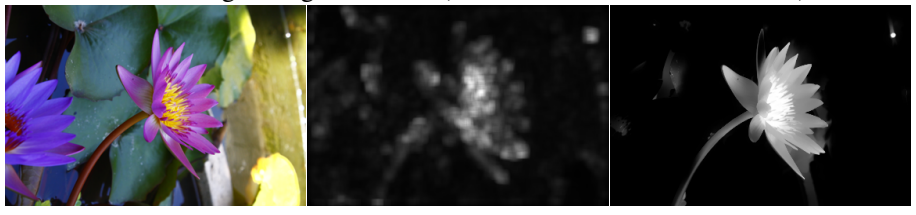

Human segmentation in 3D depth data.

(Prior consists of binary labeled ellipsoid regions given by a classifier.)

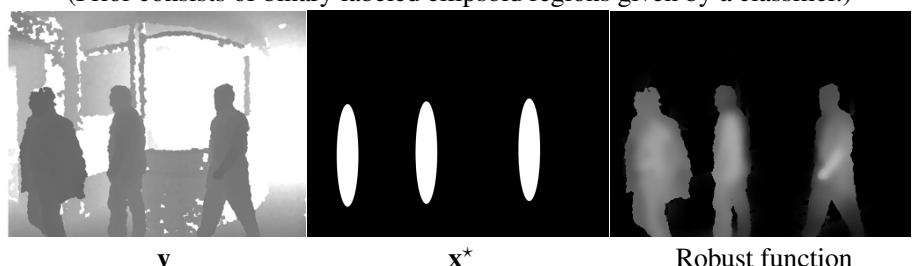

Figure 1: Applications of imposing point-wise constraints to a given prior. For each example, we show the input image structure $\mathbf{y}$, the confidence maps (labels) $\mathbf{x}^{\star}$, and our results. Segmentation results are obtained by simply thresholding and the threshold is fixed to 0.1 for all examples.

the $\mathbf{x}$. Therefore, we borrow existing principles from robust statistics [1] and adapt a robust functional to replace the least square cost as

$$
\min _{\mathbf{x}} \rho\left(\mathbf{x}-\mathbf{x}^{\star}\right)+\beta\|L \mathbf{x}\|^{2}
$$

where $\rho$ is the Huber function, which is a parabola in the vicinity of 0 and increases linearly when $\delta$ is large. Thus, the effects of large outliers can be eliminated significantly.

When written in matrix form, we use a diagonal weighting matrix $W=\operatorname{diag}\left(w_{1}, \ldots, w_{n}\right)$ to represent the Huber weight function. Therefore, the data fidelity term can be simplified as $\rho\left(\mathbf{x}-\mathbf{x}^{\star}\right)=\left\|W\left(\mathbf{x}-\mathbf{x}^{\star}\right)\right\|^{2}$. As a result, the problem Eq.(4) can be solved efficiently in an iterative least square approach. At each iteration, the optimal $\mathbf{x}$ is updated as

$$
\mathbf{x}=\left(\beta L^{\top} L+W\right)^{-1} W \mathbf{x}^{\star} .
$$

To initialize the algorithm, we could set $W=I$ for the first iteration, or when a confidence measure $m^{\star}$ is available for $\mathbf{x}^{\star}$, we could directly use $m^{\star}$ to initialize $W$. For the details of this algorithm and the comparison with other algorithms, please refer to our paper.

[1] M. Black and A. Rangarajan. On the unification of line processes, outlier rejection, and robust statistics with applications in early vision. IJCV , 19(1):57-91, 1996.

[2] L. Grady. Random walks for image segmentation. PAMI, 28(11): 1768-1783, 2006.

[3] A. Levin, D. Lischinski, and Y. Weiss. A closed-form solution to natural image matting. PAMI, 30(2):228-242, 2008.

[4] J. Shi and J. Malik. Normalized cuts and image segmentation. PAMI, 22(8):888-905, 2000. 